AnaleS Cientificos
ISSN 2519-7398 (Versión electrónica)

ARTÍCULO ORIGINAL - RESEARCH ARTICLE

http://dx.doi.org/10.21704/ac.v81i2.1701

\title{
CARACTERIZACIÓN DE UN MOTOR DE ENCENDIDO POR CHISPA, OPERADO CON BIOGÁS PARA LA GENERACIÓN DE ELECTRICIDAD
}

\section{Characterization of a spark ignition motor, operated with biogas for electricity generation}

\author{
Juvenal V. García Armas ${ }^{1 *}$ (D) \\ ${ }^{1}$ Facultad de Ingeniería Agrícola, Universidad Nacional Agraria La Molina, 15024, Lima, Perú. \\ * E-mail: juvenal@lamolina.edu.pe
}

Recibido: 20/06/2019; Aceptado: 15/12/2020; Publicado: 30/12/2020

\begin{abstract}
The objective of the study was to characterize the behavior of a spark ignition engine as an energy source, for the purpose of electricity generation, using as fuel, biogas, generated from animal excreta in a tubular biodigester, said biodegradation under anaerobic conditions resulted in a biogas composed of 59.5\% methane; $39.3 \%$ carbon dioxide; $0.2 \%$ oxygen and $1 \%$ other gases, at the outlet in the conduction pipe, filters made of steel were placed to eliminate the hydrogen sulfide. This biogas fed a $7 \mathrm{hP}$ Otto motor coupled to a $3 \mathrm{~kW}$ single-phase alternator, with a power factor of 0.7 , coupled to a $2 \mathrm{~kW}$ resistor bank, the input variables were the RPM, and the resistances, the results referred to the behavior engine for power, torque, hourly and specific consumption. They were similar in both gasoline and biogas. Differing in the magnitude developed, being less than the biogas by an average $25 \%$. Maximum values were $1500 \mathrm{~W}$ gasoline and $1250 \mathrm{~W}$ biogas. In the carbon monoxide emissions with biogas, it did not exceed $40 \mathrm{ppm}$., While with gasoline it exceeded 450ppm. Biogas is a feasible and production alternative to be used as fuel in Otto engines, for electrical or mechanical generation purposes; they also mitigate environmental pollution and the greenhouse effect.
\end{abstract}

Keywords: Otto engine; ignition; spark; generation; electricity; biodigester; biogas; environmental pollution

\section{RESUMEN}

El objetivo del estudio fue caracterizar el comportamiento de un motor de encendido por chispa como fuente de energía, con fines de generación eléctrica, empleando como combustible, el biogás, generado a partir de la excreta animal en un biodigestor tubular, dicha biodegradación en condiciones anaeróbicas dio como resultado un biogás compuesto por $59,5 \%$ de metano; $39,3 \%$ de dióxido de carbono; $0,2 \%$ de oxígeno y $1 \%$ de otros gases, a la salida en la tubería de conducción, se colocaron filtros compuestos de acero para eliminar el ácido sulfhídrico. Este biogás alimentó un motor Otto de $7 \mathrm{hP}$ acoplado a un alternador de $3 \mathrm{~kW}$ monofásico, con factor de potencia 0,7 , acoplado a un banco de resistencias de $2 \mathrm{~kW}$, las variables de entrada fueron las RPM, y las resistencias, los resultados referidos al comportamiento del motor para la potencia, par, consumo horario y especifico. Fueron similares tanto en gasolina como biogás. Difiriendo en la magnitud desarrollada, siendo menor del biogás en un 25\% promedio. Valores máximos fueron, gasolina1500W y biogás $1250 \mathrm{~W}$. En las emisiones de monóxido de carbono con el biogás, no superaba los $40 \mathrm{ppm}$., mientras que con la gasolina supera 450ppm. El biogás es una alternativa factible y de 
producción para ser usado como combustible en motores Otto, con fines de generación eléctrica o mecánica; también mitigan la contaminación ambiental y el efecto invernadero.

Palabras clave: motor Otto; encendido; chispa; generación; electricidad; biodigestor; biogás; contaminación ambiental

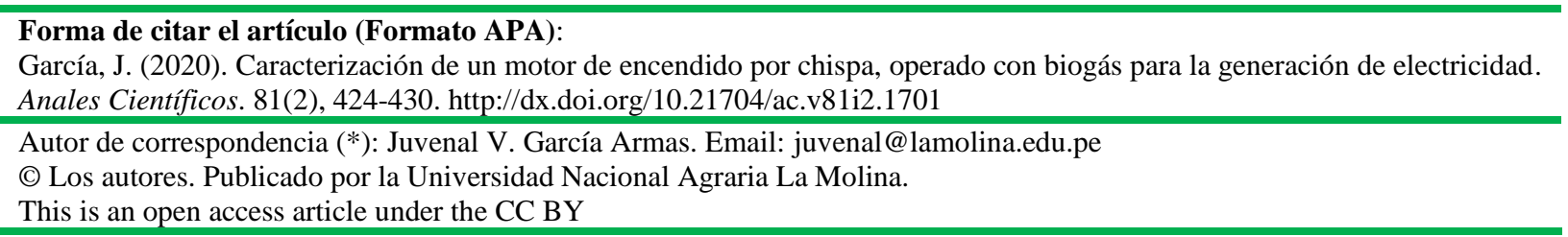

\section{INTRODUCCIÓN}

Después del Protocolo de Kyoto, el interés en el aumento de la producción de energía por medios alternativos dentro del consumo primario de energía mundial se ha ampliado significativamente con la búsqueda de la disminución en la contaminación atmosférica y por ende y la amortiguación de los cambios climáticos generados por el consumo de combustibles fósiles (Mac Lean y Lave, 2003).

En los últimos años países como Colombia y Perú, han estado trabajando en la investigación con combustibles alternativos, en la masificación del uso del gas natural vehicular, la utilización de alcohol como carburante y el desarrollo de proyectos alrededor de la biomasa, proyectos de utilización de energía eólica, proyectos de Biodiésel, entre otros. Con el fin de reducir la generación de gases de efecto invernadero.

Bajo este concepto de reducción de gases de efecto invernadero y la contaminación ambiental varios países han dedicado su trabajo de investigación en estudios que puedan reemplazar la energía proveniente de los combustibles fósiles con energías alternativas entre ellas la eólica, solar, biomasa y el biogás, esta última con buenas posibilidades en el país gracias a la gran actividad agrícola y pecuaria existente.

Desde la década de los ochenta, en Perú se ha instalado en forma puntual biodigestores para producir biogás y sobre todo un abono de excelente calidad a partir de la descomposición anaeróbica de residuos orgánicos procedentes de la explotación agropecuaria.

Por otra parte, la información existente en torno a la implementación del biogás como combustible en motores de combustión interna de encendido por chispa es mínima, en el Perú se ha encontrado más bien investigaciones en el uso del biogás para la generación de energía eléctrica con el fin de aprovechar los residuos orgánicos de la parte pecuaria (Oliveros et al., 2000), así también estudios de evaluación de motores de encendido por chispa usando como combustible el metano del biogás (Mantilla- Aguirre-Sarmiento, 2008). En Colombia se han encontrado notas técnicas sobre el procedimiento de conversión de motores de encendido por chispa para funcionamiento con el biogás como combustible (ISAT y GTZ, 2008). El biogás ha sido utilizado como combustible en motores de combustión interna encendidos por chispa desde la crisis energética en los años setenta. Numerosos estudios apuntan a que existe viabilidad técnica al utilizar este combustible como reemplazo de los combustibles fósiles. Esta viabilidad se da al comparar parámetros de operación y emisiones contra el funcionamiento de los mismos motores con combustible tradicional, en este caso gasolina (Lastra et al., 1995).

El combustible líquido tiene un mayor contenido de energía volumétrica que el gas Figura 1, y también enfría la mezcla de aire / combustible cuando se evapora en el colector de admisión. El enfriamiento produce un aumento en la densidad y, por lo tanto, la cantidad de mezcla de aire / combustible realmente absorbida en el motor en masa es mayor.

El biogás $\left(60 \% \mathrm{CH}_{4}\right)$ con un valor calorífico de $\mathrm{Hu}=25$ $000 \mathrm{~kJ} / \mathrm{nm}^{3}$ varía como un gas débil medio y provoca reducciones de potencia de aproximadamente el $20 \%$ (Mitzalff, 1998). 
Parámetros operativos de un motor de ciclo Otto. El control de potencia y velocidad del motor se realiza mediante una variación del suministro de la mezcla de aire / combustible al motor. Esto se logra mediante el funcionamiento de una válvula de mariposa situada entre el dispositivo de mezcla real y la entrada del motor. Al cerrar la válvula de mariposa se produce una caída de presión (efecto de estrangulamiento) en el flujo de la mezcla por la cual el cilindro se llena con una mezcla a una presión más baja, por lo tanto, con una menor cantidad de mezcla de aire / combustible en una base de masa y energía. Como resultado, la potencia de salida, la presión efectiva media y la eficiencia disminuyen en la operación controlada (carga parcial). El efecto de la disminución de la eficiencia Figura 1, se realiza en un aumento del combustible específico (Mitzalff, 1998).

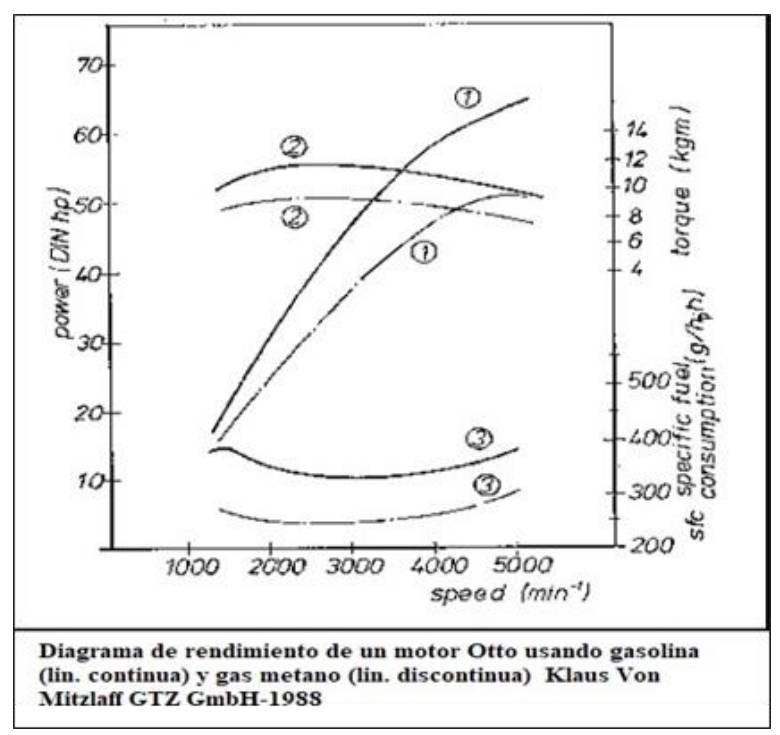

Figura 1. Curvas características de un motor Otto (K.V. Mitzalff, Engines for biogas).

Biodigestores. Producen biogás con diferentes proporciones en el contenido de metano y $\mathrm{CO}_{2}$; el porcentaje de metano está entre $40 \%$ y $70 \%$, dependiendo del material orgánico con el que se alimenta el biodigestor (Duque et al., 2006). Porcentajes aún mayores de metano, hasta $95 \%$, pueden lograrse eliminando el $\mathrm{CO}_{2}$ para de esta forma mejorar el poder calorífico del biogás. Hasta hoy, el uso final predominante de biogás ha sido el motor de combustión interna para la co-generación de electricidad y calor (Combined Heat and Power, CHP). La combustión representa un destino eficiente para biogás, en particular si hay una necesidad de energía térmica al lado del sitio de producción (Patterson et al., 2011).

Ante el enorme potencial que pudiera tener la complementación de la producción de biogás y la generación de energía eléctrica in situ, surge el objetivo de evaluar el efecto del biogás como combustible alternativo sobre los parámetros de operación del motor de combustión interna, así como la generación de monóxido de carbono como un subproducto de la combustión del biogás comparado con la operación con la gasolina.

\section{MATERIALES Y MÉTODOS}

El experimento se llevó acabo con un motor de un cilindro, cuatro tiempos, de encendido por chispa, acoplado a un alternador de $3 \mathrm{~kW}$ sus características se muestran en la Tabla 1. El motor bajo prueba estuvo equipado con un generador, y alimentaba de energía a un panel de carga de $2 \mathrm{~kW}$ formada por 20 lámparas incandescentes de 100W. La vista esquemática de la configuración experimental se representa en la Figura 2. Para medir el consumo de combustible en volumen por unidad de tiempo, se usó un depósito cilíndrico de área conocida de elaboración propia, para el gas se usó un medidor volumétrico, el tiempo fue medido con el cronómetro de un cel. pone, para la corriente y voltaje se usó un amperímetro de pinza y un voltímetro, un tacómetro para las RPM, y un medidor de gases para el monóxido de carbono todos ellos calibrados de fábrica (Tabla 2).

Los experimentos fueron realizados a diferentes velocidades de rotación del motor desde 1200 hasta los 3600 RPM en 11etapas. En cada caso se cargaron de acuerdoa lo que podía soportar el motor. El biogás se obtuvo a partir de la digestión anaeróbicade la mezcla de excretas animales, se usó el analizador LANDTEC CAM 2000 para determinar la composición del biogás 59,5\% de metano, 39,3\% de dióxido de carbono, se usó un filtro de virutas de acero para el $\mathrm{H}_{2} \mathrm{~S}$, pero no se usaron separadores de agua. El combustible líquido que se usó fue la gasolina de 90 octanos. Para todas las pruebas se dejó calentar el motor antes de realizar las mediciones con el fin de estabilizar la operación del motor. Larelación aire combustible en ningún caso fue de 15/1. La potencia, par motor, consumo de 
combustible horario, y emisiones de monóxido de carbono fueron medidos en el experimento.

Para el cálculo de la potencia se usó la fórmula: $P=I$ $\mathrm{x} V(k w)$ I en amperios y $\mathrm{V}$ envoltios.

La potencia mecánica o efectiva se tomóigual a la potencia eléctrica dado que parala carga resistiva se considera un factor de potencia unitaria.

El torque fue calculada a partir de:

$T=[(P \times 60000) /(2 \times \pi \times N)]^{*}(N . m)$
El consumo específico a partir de:

$S F C=\left(M_{f} / P\right)^{*}(k g /(k w . h))$

Tabla 1. Especificaciones del motor y generador.

\begin{tabular}{ll}
\hline \multicolumn{1}{c}{ Parámetro } & Especificaciones \\
\hline Tipo de motor & 1 Mono cilindro, 4 tiempos, \\
Potencia de motor & $5,2 \mathrm{~kW}$ (7Hp) \\
Enfriamiento & Por aire \\
Cilindrada & $315 \mathrm{cc}$ \\
Pot. Max. generador & $3,5 \mathrm{kw}$ \\
Potencia nominal & $3 \mathrm{kw}$ \\
\hline
\end{tabular}

Tabla 2. Características de los instrumentos de medida.

\begin{tabular}{lllllr}
\hline \multicolumn{1}{c}{ Instrumento } & \multicolumn{1}{c}{ Marca } & \multicolumn{1}{c}{ Origen } & \multicolumn{1}{c}{ Modelo } & \multicolumn{1}{c}{ Rango } & \multicolumn{1}{c}{ Exactitud } \\
\hline Tacómetro & Monarch & U.S.A. & PLT200 & $5-200000 \mathrm{rpm}$ & $0,01 \%$ \\
Analizador $\mathrm{CH}_{4}$ & LANDTEC & UK & CAM 2000 & Auto & $0.3 \%-0,5 \%$ \\
Analizador de CO & CEM & U.S.A. & CO180 & $0-100 \% \mathrm{CH}_{4}$ & $0.01 \%$ \\
Voltímetro & Konstar & China & YX-360 TR & $0-1000$ volt. & $0,10 \%$ \\
Amperímetro & SUNWA & China & DT-266 & $0-1000$ Amp. & $0,10 \%$ \\
\hline
\end{tabular}

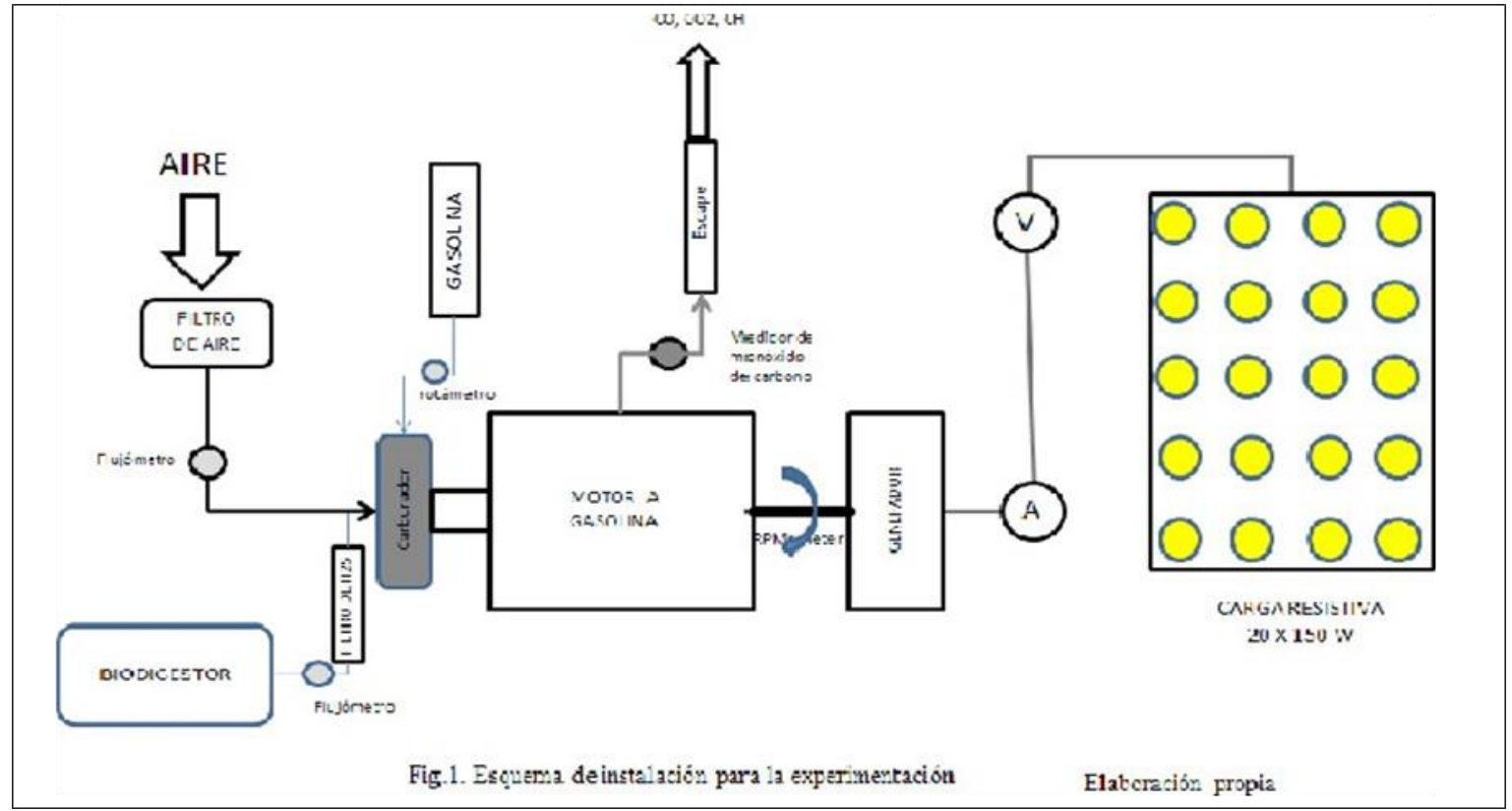

Figura 2. Configuración experimental del sistema de prueba.

\section{RESULTADOS Y DISCUSIÓN}

\section{De la potencia del motor}

En la Figura 3 observa que la potencia desarrollada por el motor crece mientras los rpm aumentan (Mitzalff, 1988), Figura 1. Con la gasolina la potencia máxima es de 1520 W a 3000 RPM, con el biogás la potencia máxima es de $1250 \mathrm{~W}$ a $3300 \mathrm{rpm}$. La diferencia en el rendimiento está entre el 20 y $30 \%$ esto se debió a que el biogás no está constituido al $100 \%$ de pureza como en el caso de la gasolina sino como en nuestro caso el metano que es el combustible solo corresponde al 
59,5\% más aún si se tiene presente que el $\mathrm{CO}_{2}$ retarda la velocidad de la flama (Porpatham et al., 2007) quien encontró que la reducción en la concentración de $\mathrm{CO}_{2}$ en el biogás conduce a una mayor eficiencia y salida de potencia en los motores de encendido por chispa. Si mejoráramos el contenido de metano en el biogás que es lo mismo que eliminar $\mathrm{CO}_{2}$ obtendríamos mayor eficiencia en el rendimiento del motor (Bari, 1996). Lo que si es necesario recalcar es que el comportamiento del motor con ambos combustibles sigue el mismo patrón (Figura 1).

\section{Del torque o par motor}

El torque es la capacidad que tienen los motores de responder a las solicitudes de esfuerzo extra en determinados momentos.

En la Figura 4 se observa la tendencia de la curva resultante de la operación del motor a gasolina, así como la operación con biogás, típicas y el valor máximo de torque con biogás es de 4,7 N-m a $1220 \mathrm{rpm}$, y con la gasolina el torque máximo es de 6,27 N-m, a 1220 $\mathrm{rpm}$. Es normal que las torques más altas se presenten a bajas revoluciones y conforme va en aumento la velocidad de giro del motor esta disminuya. Es de interés que la presiones que ejercen los gases quemados a partir del metano también ejercen fuerzas considerables que hacen posible altos torques que son suficientes para accionar generadores eléctricos u otras máquinas.

\section{Del consumo horario de combustible}

La Figura 5 muestra que cada curva sigueel patrón según las curvas característicasde un motor (Mitzalff, 1988), así mismo se observa que el consumo volumétrico para elbiogás es mayor que el de gasolina para todoel rango de revoluciones de funcionamiento del motor, esto debido a que el gas estáa una presión ligeramente superior a la atmosférica, además de tener solamente el $60 \%$ de pureza siendo el resto dióxido de carbono. Siendo para la gasolina volúmenesinferiores al litro dado que el suministro es líquido.

\section{Del consumo específico de combustible}

En la Figura 6 que la curva del biogás y de la gasolina presenta la misma tendencia, condisminución de valor hacia el punto de mayor potencia. En ese caso 0,36 $\mathrm{kgr} / \mathrm{kW}$-h a $3000 \mathrm{rpm}$ para el caso de la gasolina y de $5,9 \mathrm{~m}^{3} / \mathrm{kW}$-h a $3000 \mathrm{rpm}$ para el caso del metano. Estos valores son los que nos indican si un motor es más eficiente que otro, así como también si el combustible que se está usandoproduce más energía por unidad de masa eneste caso la gasolina está desarrollando más potencia por unidad de masa.

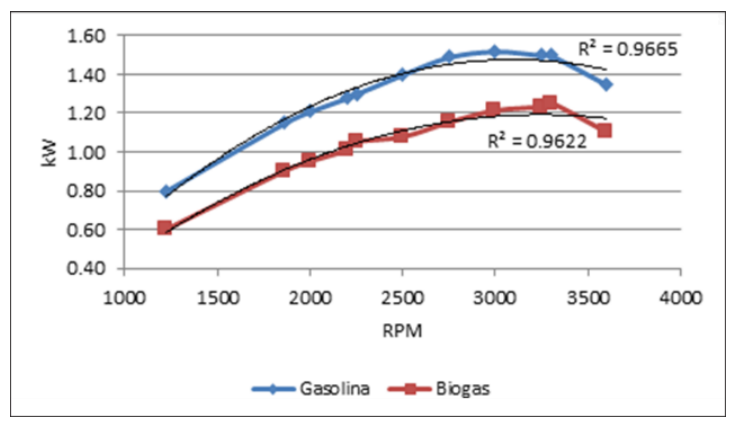

Figura 3. Curvas de potencia desarrolladas por el motor de combustión interna.

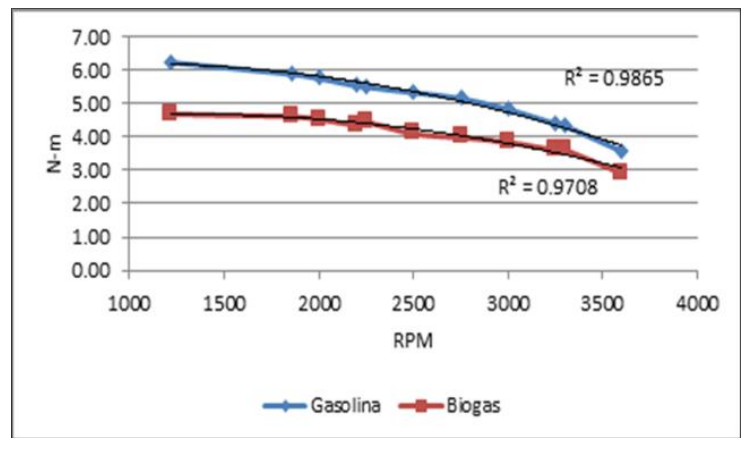

Figura 4. Curvas de Torque desarrolladas por el motor de combustión interna.

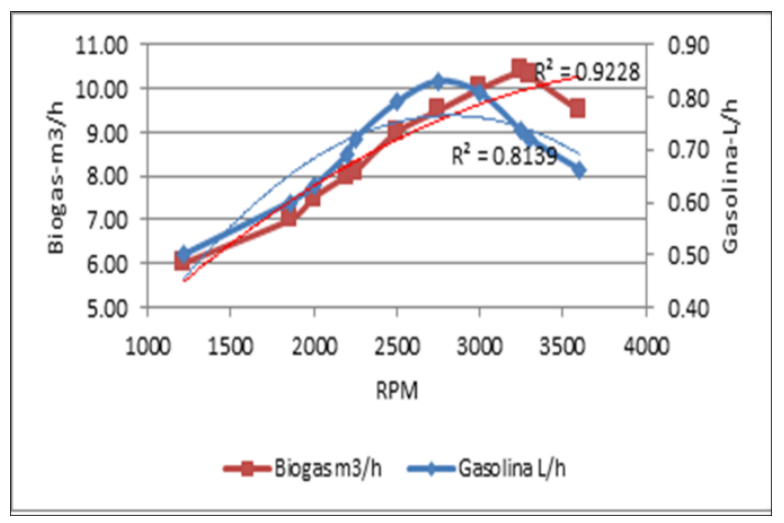

Figura 5. Curvas de Consumo hora desarrolladas por el motor de combustión interna.

\section{De las emisiones de CO}

Los valores de contenido de monóxidode carbono sobrepasan las $800 \mathrm{ppm}$ a los 3600RPM cuando se 
opera el motor con gasolina mientras que al ser operados con biogás los valores bajan a 39 ppm, por lo quepodemos afirmar que el operar el motor en base a biogás va a mitigar los efectos de la contaminación ambiental, comparando estos valores podemos aseverar que el uso de biogás colabora con el cuidado ambiental.

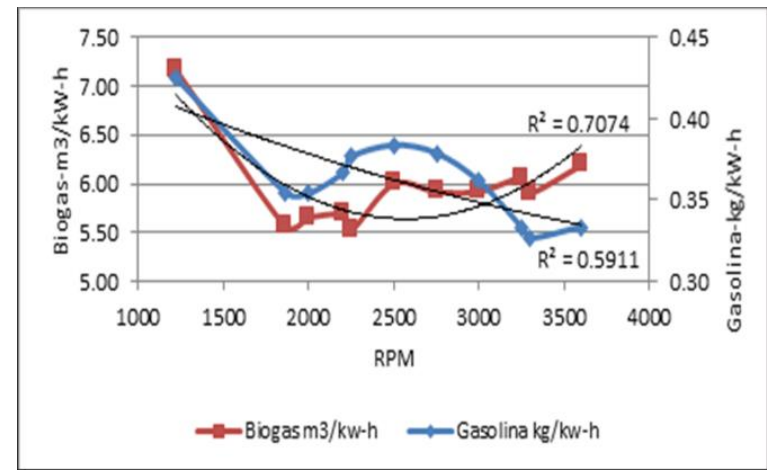

Figura 6. Curvas de Consumo específico del motor de combustión interna.

\section{CONCLUSIONES}

Las características de operación de un motor de combustión interna de encendido por chispa son prácticamente iguales tanto en la operación con gasolina y biogás, siendo de la misma tendencia en ambos casos. Así mismo la potencia desarrollada con respecto al biogás difiere en un $25 \%$ en promedio debido a la disminución en el poder calorífico del biogás y su alto contenido de $\mathrm{CO}_{2}$. La producción de biogás en situ es factible y su implementación fácil a nivel depequeñas granjas en el área rural debido a la presencia de materia prima in situ como es elcaso de porcinos, vacunos y aves. Además, su conversión y uso toma importancia al tratarse de asuntos de mitigación del cambio climático. Se recomienda que, esnecesario investigar las posibilidades de desarrollar filtros simples para el $\mathrm{H} 2 \mathrm{~S}$, así como separadores de $\mathrm{CO}_{2}$ de bajo costo paramejorar la calidad del biogás. También, es necesario proponer políticas para el uso de biodigestores con fines de generación de energía en zonas rurales con potencial de producción de biogás. Ya sea a partir deexcretas de animal o de las aguas servidas evitando así la contaminación ambiental en especial de los ríos.

\section{Conflictos de intereses}

Los autores firmantes del presente trabajo de investigación declaran no tener ningún potencial nflicto de interés personal o económico con otras personas $u$ organizaciones que puedan influir indebidamente con el presente manuscrito.

\section{Contribuciones de los autores}

Preparación y ejecución: JGA; Desarrollo de la metodología: JGA; Concepción y diseño: JGA; Edición del artículo: JGA; Supervisión del estudio: JGA

\section{LITERATURA CITADA}

- Bari, S. (1996). Effect of carbón dioxide on the performance of biogás/diésel duel-fuel engine. Renew Energy, 9(1-4): 1007-10.

- European Community. (2009). Directive 2009/28/EC. On the promotion of theuse of energy from renewable sources and amending and subsequently repealing Directives 2001/77/EC and 2003/30/EC.2009.04.23. Off J EU L140, $16 \mathrm{e} 62$.

- ISAT, GTZ. (2015). Biogás Digest. 1. Disponible en: $\quad$ www.gtz.de/de/dokumente/en biogasvolume1. pdf, consultado el 14de marzo de 2015.

- Brizio, E. (2012). Ecosustainable Biomethaneand Fertilizer Production through Anaerobic Codigestion of Animal Manure and Energy Crops. PHD thesis. Politecnico di Torino.

- Duque, C., Galeano, C. \& Mantilla, J. (2006). Plug flow biodigester evaluation., Livestock Research for Rural Development, 18(04).

- Lastra, L.L., Valderrama, A., Vera, E. \& Amezquita, F. (1995). Experimentacióny cálculo de Motores de Combustión Interna" Publicación. Universidad Nacional de Ingeniería- Instituto de Motores de Combustión Interna-UNILima.

- Mantilla, B., Aguirre, L. \& Sarmiento, J. (2008). Evaluación experimental de un motor encendido por chispa que utiliza biogás como combustible. revista ingeniería e investigación,28(2):(131-141).

- MacLean, H.L. \& Lave, L.B. (2003). Evaluating Automobile Fuel/Propulsion System Technologies. Progress in Energyand Combustion Science, 29: 169. https://doi.org/10.1016/S03601285(02)00032-1 
- Mitzalff, K. (1988). Engines for biogás, GTZ, GmbH 1988.

- Mustafi, M., Raine, R. \& Bansal, P. (2006). The use of biogas in internal combustion engines: a review. in: Proceedings of the Spring Technical Conference of the ASME Internal Combustion Engine Division, USA.

- Oliveros, A., Lira, R. \& Figueroa. (2000). eneración de energía eléctrica a partir de biogás, DOI: https://doi. org/10.21754/tecnia.v10i2.457
- Patterson, T., Esteves, S., Dinsdale, R. \& Guwy, A. (2011). Life cycle assessment of biogas infrastructure options on a regional scale. Bioresour. Technol., 102: 7313e7323.

- Porpatham, E., Ramesh, A. \& Nagalingam, B. (2007). Investigation on the effect of concentration of methane in biogas when used as a fuel for a spark ignition engine, 87: 1651-1659. 